\title{
Up-regulation of human immune system function by Donkey's Milk
}

\author{
Saeid Taghiloo ${ }^{1,2 \dagger}$, Esmaeil Allahmoradi ${ }^{1,2 \dagger}$, Seyedeh Forough Sadeghian-Kiadehi ${ }^{1}$, \\ Versa Omrani-Nava ${ }^{1,2}$, Eisa Nazar ${ }^{3}$, Mohammad Ali Ebrahimzadeh ${ }^{\circledR *}$
}

\author{
${ }^{1}$ Department of Immunology, School of Medicine, Mazandaran University of Medical \\ Sciences, Sari, Iran, ${ }^{2}$ Student Research Committee, Mazandaran University of Medical \\ Sciences, Sari, Iran, ${ }^{3}$ Department of biostatistics and epidemiology, Mashhad University \\ of Medical Sciences, Mashhad, Iran, ${ }^{4}$ Pharmaceutical Sciences Research center, \\ Hemoglobinopathy Institute, Mazandaran University of Medical Sciences, Sari, Iran
}

\begin{abstract}
Donkey's milk represents a good alternative to human milk because of its chemical characteristics similar to those of human's. In present study, the pro- and anti-inflammatory effects of donkey's milk were evaluated on peripheral blood mononuclear cells (PBMCs). PBMCs were isolated from 12 young and 12 aged normal subjects. PBMCs were cultured with or without the optimal and noncytotoxic dose of pasteurized donkey's milk, and polymyxin B was used to inhibit the possible endotoxin contamination. Following 18 hours incubation, culture supernatants were harvested to measure the secreted Tumor necrosis factor- $\alpha$ (TNF- $\alpha$ ), Interleukin-6 (IL-6), Interleukin-8 (IL-8) and Interleukin-10 (IL-10) by ELISA. Donkey's milk significantly increased TNF- $\alpha(\mathrm{p}=0.01)$, IL-8 $(p<0.0001)$, IL-6 $(p<0.0001)$ and IL-10 $(p=0.01)$ levels in PBMCs. In addition, the levels of IL-6 $(p=0.002)$, IL-8 $(p=0.002)$ and TNF- $\alpha(p=0.002)$ from aged subjects were significantly higher compared with young subjects. In contrast with these data, the level of IL-10 was markedly reduced from aged subjects $(p=0.02)$. Considering the immune-potentiation effects of donkey's milk, it is suggested investigating milk as a beneficial dietary component for up-regulating the immune response in aged people.
\end{abstract}

Keywords: Donkey's milk. ELISA. Immune system. Aged subject. Cytokine.

\section{INTRODUCTION}

Immune senescence is a physiological phenomenon of aging, which declines the defense power resulting in infection, cancer and immune-related diseases (Ginaldi et al., 2001). Failure to produce CD4+ T-cells, decreased natural killer (NK) cell cytotoxicity, poor proliferative responses to antigenic stimulation and impaired cytokine production are among the outstanding features

*Correspondence: M. A. Ebrahimzadeh, Department of Medicinal Chemistry, School of Pharmacy, Mazandaran University of Medical Sciences, 18th km. Bolvarkhazar, Sari, 48189, Iran. Tel.: +98 151 3543081-3; Fax: +98 151 3543084. Email: zadeh20@gmail.com. † Saeid Taghiloo and Esmaeil Allahmoradi have contributed equally to this study. of this event (Mocchegiani, Malavolta, 2004; Linton, Lustgarten, Thoman, 2006).The use of natural products has been traditionally considered for preventing the diseases and strengthening the immunity. In this context, several studies have been carried out on immune modulatory compounds (Das, Bordoloi, Newar, 2014). One of these natural products that have recently received much attention is Donkey's milk.

Although little effort has been paid to donkey's milk in the past, in the last years it has been the subject of intense research because of its similar composition to human milk (Jirillo, Jirillo, Magrone, 2010). Interest in donkey's milk for human nutrition is increasing because of its distinctive composition and unique functional 
properties (Lionetti et al., 2012). Nowadays, in some countries, especially in European ones, donkey's milk is available on the marketplace and is mainly targeted to newborns as well as to elderly and convalescent people (Amati et al., 2010). In particular, it is a good alternative, in cases of allergy to cow's milk and lack of breastfeeding (Jirillo, Jirillo, Magrone, 2010). Donkey's milk compared with other sources of milk contains more lysozyme and immunoglobulins, and its lower ratio of casein/whey seems to be a major factor in fast digestion and hypo-allergic features (Carroccio, Cavataio, Iacono, 1999). One of the main allergens in children is $\beta$-lactoglobulin. Although it is the major whey protein in cow's milk, it is absent in human milk (Carroccio, Cavataio, Iacono, 1999; Miranda et al., 2004). In donkey's milk, the content of $\beta$-lactoglobulin is approximately $40 \%$ of the whey proteins equal at the level in mare milk and lower than that in cow milk (Miranda et al., 2004). This condition may be related to the hypoallergenic characteristic of donkey's milk (Businco et al., 2000; Carroccio et al., 2000). The mechanism for tolerance may be related to the specific levels of the major allergenic components in the milk. High concentrations of lactoferrin and lysozyme are responsible for antimicrobial properties against potential gastrointestinal pathogens such as E. coli and L. monocytogenes (Vincenzetti et al., 2008). This issue is beneficial in children or elderly, because of their weaker immune system (Vincenzetti et al., 2008; Jirillo, Jirillo, Magrone, 2010; Tidona et al., 2011).The concentration of lysozyme in human milk increases strongly after the second month of lactation, suggesting that this enzyme plays an important role in fighting infections in breastfed infants during the late lactation (Uniacke-Lowe, Huppertz, Fox, 2010). Donkey's milk is a rich source of polyunsaturated fatty acids (PUFA), especially n-3 and contains lower PFUA n-6 (Gastaldi et al., 2010).The effects of PFUA n-3 on immune modulation is through the synthesis of lipid mediators and cytokines. N-3fatty acids play an important role in cardiovascular health and the prevention of inflammation and autoimmunity (Vincenzetti et al., 2008). Diets, including donkey's milk reduce the inflammatory symptomsand, increase energy levels and detoxification in treated animals, which may be due to higher levels of PUFA than in cow's milk (Gastaldi et al., 2010; Lionetti et al., 2012).

Thus, it seems that the role of donkey's milk in stimulating or inhibiting inflammatory responses requires further evaluation. Considering that the appropriate stimulation of the immune system is important in aged people, the effect of donkey's milk on their immune response can be useful in future therapeutic applications. In the present in vitro study, the pro- and anti-inflammatory effects of donkey's milk were evaluated on human peripheral-blood mononuclear cells (PBMCs).

\section{MATERIAL AND METHODS}

\section{Preparation of milk samples}

Milk from donkey located in Zanjan, northwest Iran, was used for this study. Donkey's milk samples were collected and kept in an ice box at less than $4{ }^{\circ} \mathrm{C}$ during transportation for about one hour. After arrival, samples were pasteurized at $63{ }^{\circ} \mathrm{C}$ for 30 minimums and stored at $-70{ }^{\circ} \mathrm{C}$ until analysis.

\section{Study subjects}

A total of 12 young subjects (six males and six females; mean age 24.33 years) and 12 aged subjects, including six males and six females with mean age 54.00 years were included within this study. Before blood sampling, each subject was clinically evaluated in order to exclude concurrent diseases or drug assumption, which may affect the immune responsiveness. Therefore, based on these criteria, only healthy people were included within this study, after obtaining written informed consent according to the Ethical Committee of Mazandran University of Medical Sciences. After that four milliliters of heparinized peripheral blood were taken from each study subject. The demographic characteristics of study subjects are summarized in Table I.

\section{Isolation of peripheral blood mononuclear cells}

The fresh peripheral blood mononuclear cells (PBMCs) from study subjects were isolated by density gradient centrifugation on Ficoll-Histopaque (Biosera, Nuaille, France). Isolated PBMCs were washed twice with RPMI- 1640 culture medium (Biosera, Nuaille, France), and re-suspended in the same medium supplemented with penicillin $(100 \mathrm{IU} / \mathrm{mL})$, and streptomycin $(100 \mu \mathrm{g} / \mathrm{mL})$ (Biosera, Nuaille, France). The viability of obtained cells was $95 \%$ as determined by exclusion of Trypan blue viability test. 
TABLE I - Demographic characteristics of study subjects

\begin{tabular}{lcr}
\hline Characteristics & Young subjects & Aged subjects \\
\hline Sex & \multicolumn{1}{c}{} \\
Male & 6 & 6 \\
Female & 24.33 years & 6 \\
\hline Age $($ years $)$ & $21-27$ years & 54 years \\
Mean \pm SD & & $47-62$ years \\
Range & $8375 \pm 434$ & \\
\hline White blood cell count $(/ \mu \mathrm{L})$ & $7210-9790$ & $6485 \pm 704.1$ \\
Mean \pm SD & & $4200-8840$ \\
Range & $5.28 \pm 0.18$ & $4.70 \pm 0.19$ \\
\hline Red blood cell count $(/ \mu \mathrm{L})$ & $4.42-5.74$ & $4.02-5.42$ \\
Mean $\pm \mathrm{SD}\left(\times 10^{6}\right)$ & & $12.42 \pm 0.24$ \\
Range $\left(\times 10^{6}\right)$ & $13.97 \pm 0.72$ & $11.30-13.00$ \\
\hline Hemoglobin $(\mathrm{g} / \mathrm{dL})$ & $11.50-15.60$ & 222000 \\
Mean $\pm \mathrm{SD}$ & & $205000-260000$ \\
Range & 222000 &
\end{tabular}

\section{Cell culture and stimulations}

In order to evaluate the effects of donkey's milk on cytokine production from PBMCs, $2 \times 105$ cells were cultured in $200 \mu \mathrm{L}$ RPMI-1640-L-glutamine medium (Biosera, Nuaille, France) supplemented with $10 \mu \mathrm{L}$ of donkey's milk, 100IU/ml penicillin, $100 \mu \mathrm{g} /$ $\mathrm{ml}$ streptomycin (Biosera, Nuaille, France), and 10\% heat inactivated fetal calf serum (FCS) in flat-bottomed 96-well microplates. Before started experiments, the optimal and non-cytotoxic dose of pasteurized donkey's milk were determined. Polymyxin B (PMB-50 $\mu \mathrm{g} /$ $\mathrm{mL}$, Invitrogene, California, USA) was added to all wells to inhibit the possible endotoxin contamination. Lipopolysaccharide (LPS-100 pg/mL, Invitrogene, California, USA) was also applied as positive control. All cultures were performed in triplicate and plates were incubated at $37{ }^{\circ} \mathrm{C}$ in a humidified atmosphere with $5 \% \mathrm{CO} 2$ for 18 hours. The culture supernatants were harvested and stored at $-20{ }^{\circ} \mathrm{C}$ until use for ELISA cytokine assay.

\section{Cytokine assay}

The amount of differentiation-inducing factors of interleukin (IL)-6, IL-8, IL-10, and tumor necrosis factor (TNF)- $\alpha$ in the cultured supernatant, before and after treating with donkey's milk was measured at a wavelength of $450 \mathrm{~nm}$ by an Enzyme-linked immunosorbent assays (ELISA) kit, as described by the manufacturer's protocol (Sanquin, Amsterdam, The Netherlands). Briefly, 96-well plates (SPL, South Korea) were coated overnight with coating antibody of IL-8, IL-6, IL-10, and TNF- $\alpha$ in phosphate buffer saline (PBS, 0.15M). The plate was then washed (PBS plus $0.05 \%$ Tween-20(PBS-T)). All samples of interestedwere added in the wells diluted in PBS-T. Next, polyclonal biotinylated anti-IL-8, -IL-6, -IL10 , and anti-TNF- $\alpha$ were simultaneously added to each well, and incubated at room temperature on a shaker. The plate was washed again and incubated with streptavidin-poly HRP (horseradish peroxidase). Finally, the plate was washed and incubated with 
substrate solution. The absorbance was measured at $\mathrm{OD} 450 \mathrm{~nm}$ by a microplate spectrophotometer (Synergy H1 Hybrid Reader). Samples were run in quadruplicate, followed by normalizing IL-8, IL6 , IL-10, and TNF- $\alpha$ values to the measured protein concentration to compare all cell types.

\section{Statistical analysis}

Statistical analyses were performed with Graph Pad Prism 6 and SPSS20 statistic's software. Data are expressed in the mean \pm standard error of the mean (SEM). Nonparametric Mann-Whitney U-test was used to calculate the significance difference between the groups. For comparison of three groups, the Kruskal-Wallis test was used, and Wilcoxon test was used for comparison between the individuals before and after the treated milk group. $\mathrm{P}<0.05$ was considered statistically significant.

\section{RESULTS AND DISCUSSION}

\section{Polymixin B inhibited LPS for stimulate cytokine secretion}

Currently, investigation on the properties of donkey's milk has become more popular than in the past, because it contains other factors that may be beneficial to human health (Lionetti et al., 2012). Donkey's milk has recently been considered as a good alternative to a newborn because, its nutrient composition, particularly the proteins, is very similar to human milk (Monti et al., 2007; Criscione et al., 2009). Some components of donkey's milk possess unique characteristics such as bactericidal properties (Vincenzetti et al., 2008). Literature reports that donkey's milk has a stronger anti-microbial activity than other types of milk against different microorganisms (Vincenzetti et al., 2008; Tidona et al., 2011). Moreover, it has remarkable antimicrobial characteristics due to the high concentration of lysozyme, lactoferrin and further natural inhibitory elements, which confer high hygienic potentials (Salimei et al., 2004; Zhang et al., 2008). In addition, lysozyme has other physiological functions, including inactivation of certain viruses, immuno-regulatory activity, anti-inflammatory, and anti-tumor activity (Tidona et al., 2011). In Italy, donkey's milk is mainly used for newborn nutrition as a natural substitute milk when breast-feeding is not possible.It also is used as a replacement to bovine milk in diet therapy of patients affected by cow's milk protein allergy (CMPA) (Jirillo, Jirillo, Magrone, 2010; Pilla et al., 2010).

First, we used polymyxin B (PMB) to inhibit the possible endotoxin contamination in donkey's milk. For this purpose, the optimal dose of LPS, PMB were determined using serial dilution, and the best of point were chosen. Herein, we stimulated PBMCs with LPS (100 pg/mL) and evaluated IL-6, IL-8, IL-10, and TNF- $\alpha$ in the presence or absence of PMB (50 $\mu \mathrm{g} / \mathrm{mL}$ ) by ELISA. Consistent with this hypothesis, we observedthat the levels of IL-6, IL-8, and TNF- $\alpha$ markedly reduced in stimulated cells with LPS in the presence of PMB compared with absent from PMB (Figure 1A-C). In contrast, no differences were seen at the levels of IL-10 the presence or absence of PMB (Figure 1D).

\section{Increased cytokine levels from PBMCs treated by donkey's milk}

Over recent years, the immune properties of donkey's milk have shown their potential exploitation in human nutrition (Tafaro et al., 2007). In the present study, the alternation of IL-8, IL-6, TNF- $\alpha$, and IL-10 from PBMCs stimulated with donkey's milk compared to un-stimulated cells from both young and aged subjects has been determined, also the optimal dose of donkey's milk for cell culture was determined by cytokine assay. Consistent with our hypothesis, we observed that the level of IL-8, a neutrophil chemo attractant, and IL-6, a protective acute phase protein was significantly higher by treated donkey's milk than un-treated. Additionally, donkey's milk could induce a relatively moderate increase in cytokine production of TNF- $\alpha$ from PBMCs, suggesting a low pro-inflammatory profile in these subjects. Similarly, normal human PBMCs stimulated with donkey's milk release IL-10 in their supernatants, indicating a certain degree of regulatory effect, and maintaining immune homeostasis in the host (Figure 2C, D). These cytokines are contributory to an immune response and result in maturation, differentiation and regulation of immune cells for defense mechanisms of the host (Jirillo, Magrone, 2014). Previous studies have indicated that donkey's milk produces nitric oxide (NO) from PBMCs and induces adaptive immunity as evidenced by cytokine production (Jirillo, Jirillo, Magrone, 2010). 


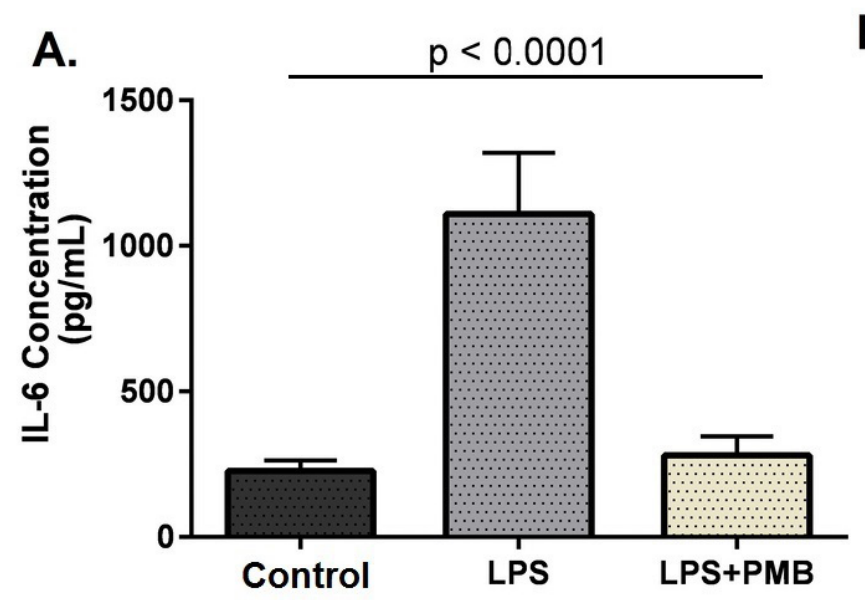

B.

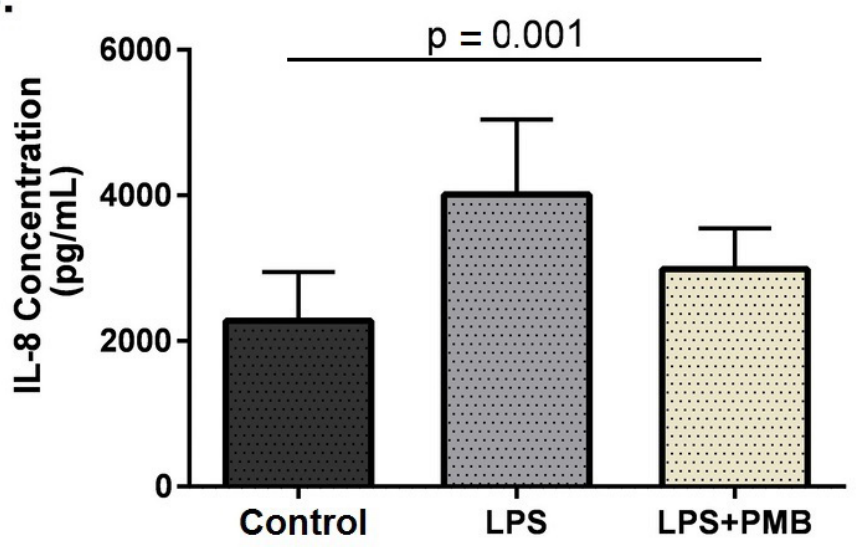

C.

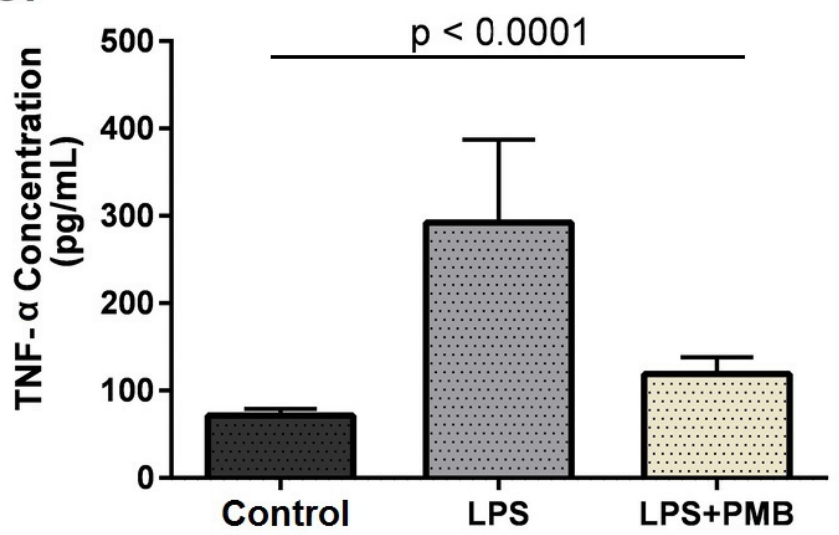

D.

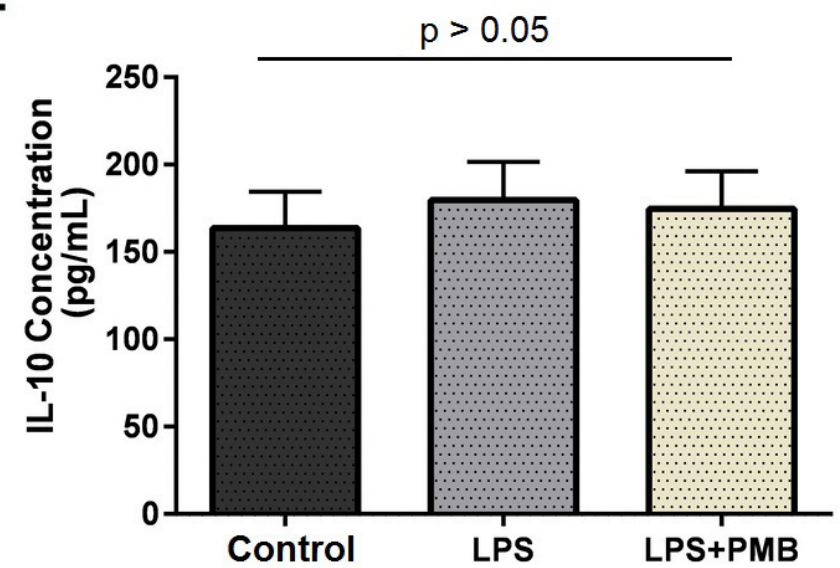

FIGURE 1 - Inhibition of Lipopolysaccharide (LPS) by polymyxin B (PMB). PBMCs isolated from study subjects (by ELISA). (A) IL-6 levels, (B) IL-8 levels, (C) TNF- $\alpha$ levels, and (D) IL-10 levels from study subjects. (A-D) The results are represented as Mean \pm SEM. Statistical comparisons made by Kruskal-Wallis test, differences were considered statistically significant for $\mathrm{p}<0.05$. 
A.

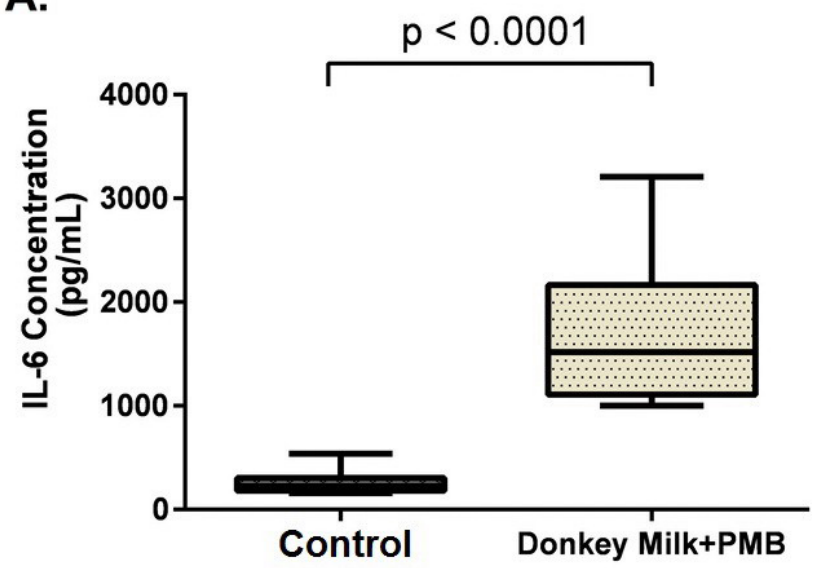

C.

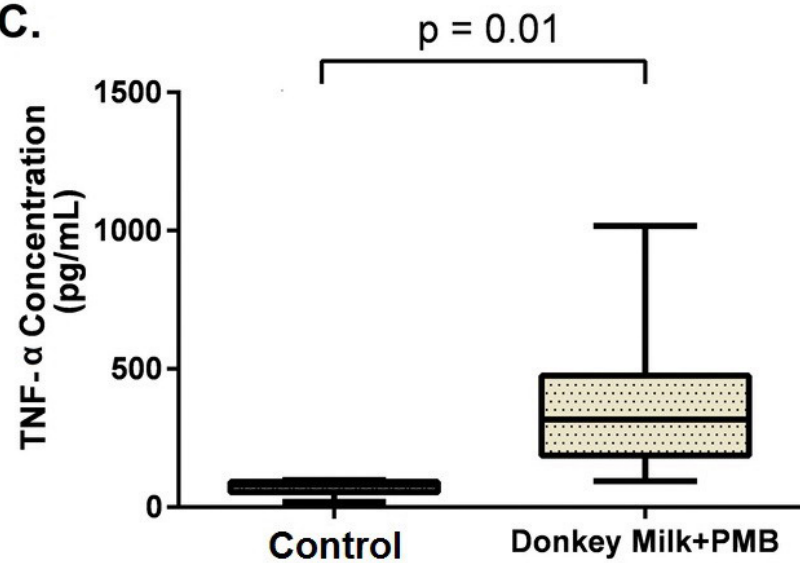

B.

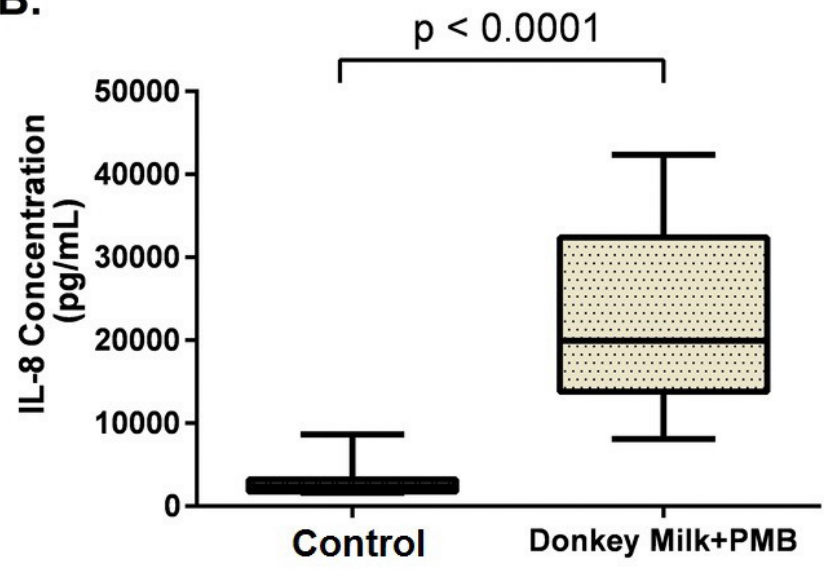

D.

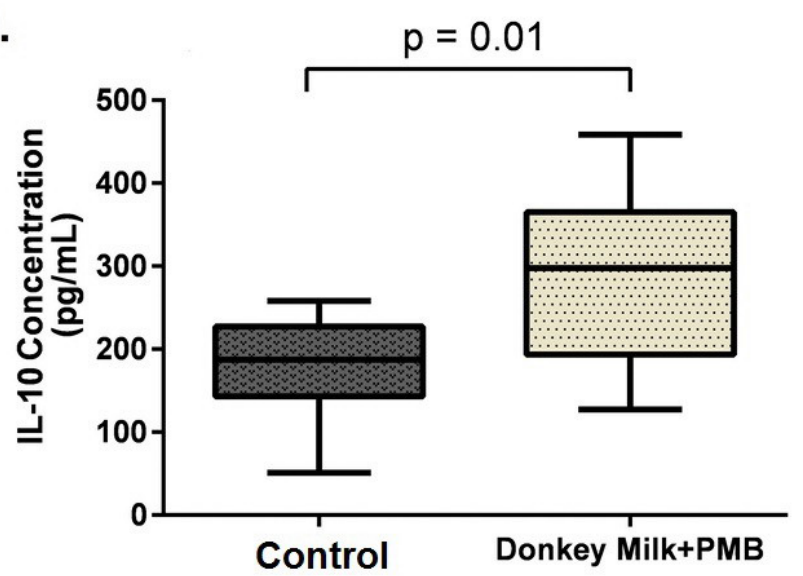

FIGURE 2 - Cytokine levels from PBMCs with or without treated by donkey's milk in study subjects (by ELISA). (A) IL-6 levels, (B) IL-8 levels, (C) TNF- $\alpha$ levels, and (D) IL-10 levels from subjects with or without treatment. (A-D) Horizontal bars, boxes and whiskers represent median, $25 / 75 \%$ quartiles and range and the lines show the most extreme observations. Statistical comparisons made by Wilcoxon matched-pairs test, differences were considered statistically significant for $\mathrm{p}<0.05$. 

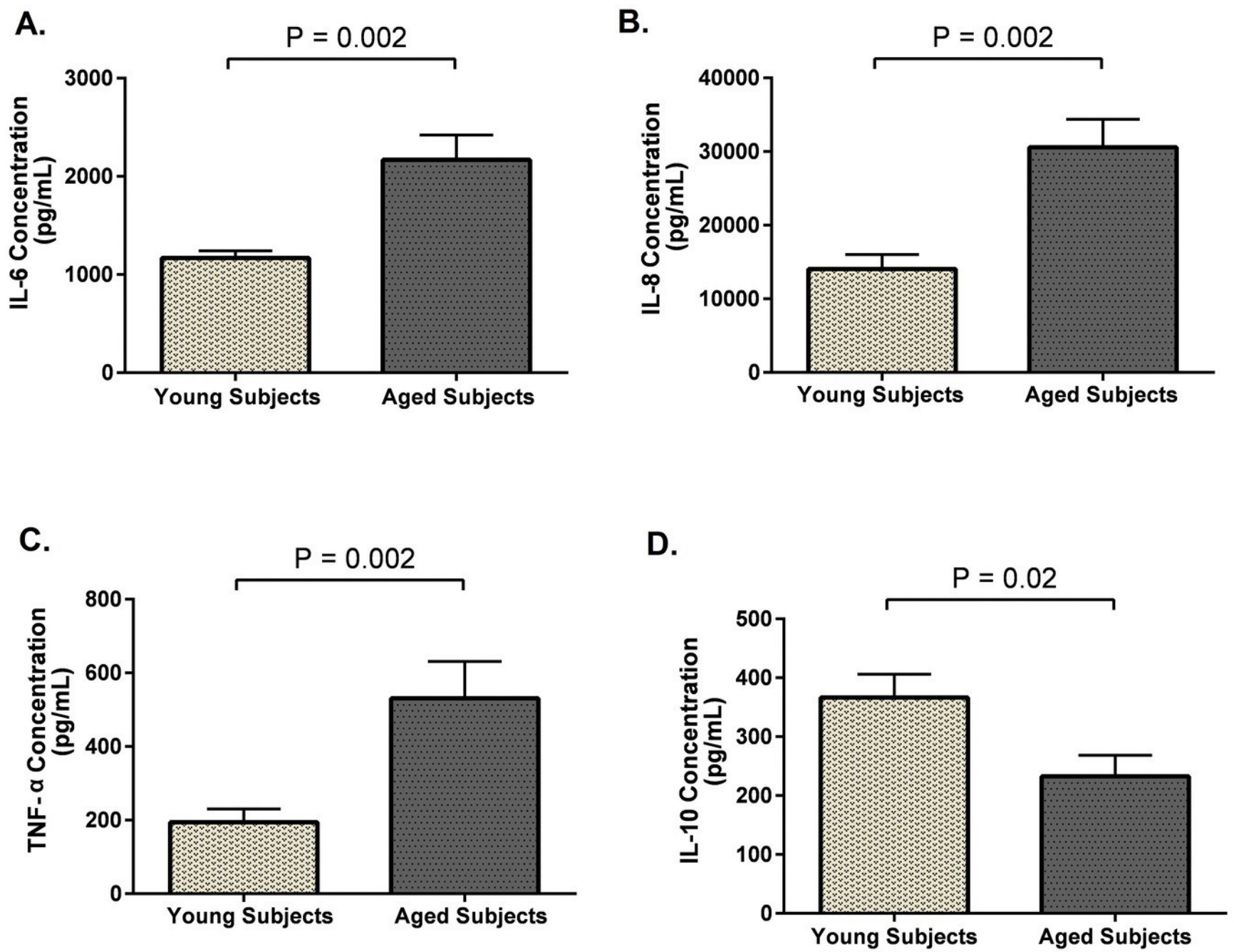

FIGURE 3 - Cytokine production from PBMCs treated by donkey's milk in young subjects and aged subjects (by ELISA). (A) IL-6 levels, (B) IL-8 levels, (C) TNF- $\alpha$ levels, and (D) IL-10 levels from subjects. (A-D) The results are represented as Mean \pm SEM. Statistical comparisons made by Mann Whitney test, differences were considered statistically significant for $\mathrm{p}<0.05$. 


\section{Improved cytokine production from PBMCs treated by donkey's milk in aged subjects compared to young subjects}

Another study has previously shown that donkey's milk could promote the cytokine potentiating response of precursor T-cells and macrophages after specific recognition of tumor cells, which explains the pathway and the possible immune mechanism of donkey's milk (Mao et al., 2009). Apart from the above mentioned properties, donkey's milk is receiving increasing attention because of other interesting biological activities, such as the antioxidant activity, the immunestimulating ability, and anti-inflammatory effects, which may be useful for the treatment of immunerelated diseases in humans and prevent atherosclerosis (Tafaro et al., 2007; Piovesana et al., 2015). Moreover, other interesting activities have been reported, such as the antiviral activity, and the anti-proliferative effect on A549 human lung cancer cells (Mao et al., 2009; Sadiq et al., 2013).

Immuno-senescence is characterized by a progressive decline of immune functions with age and severe impairment of both innate and adaptive immune responses (Jirillo et al., 2008). This fact seems to be accounted for the increased susceptibility of aged people to infections, tumors and autoimmune diseases (Linton, Lustgarten, Thoman, 2006). Nutrition seems to be fundamental in the mechanism of immune recovery in the elderly (Jirillo et al., 2008). Based on these observations, we aim to investigate the association between donkey's milk and immunepotentiation in aged subjects. Herein, the results of the present experiment showed that the levels of IL-8, IL6 , and TNF- $\alpha$ were significantly higher from PBMCs stimulated with donkey's milk in aged subjects than young subjects. In contrast to our findings, the concentration of IL-10 was markedly reduced from aged subjects when compared to young subject. In agreement with our results, a previous study reported that, the levels of pro-inflammatory cytokines such as TNF- $\alpha$ and IL- 6 were increased from aged subjects after intake donkey's milk compared to before intake milk during one month (Amati etal., 2010). These data represent an important understanding of the actions in vitro of donkey's milk that may potentially be useful as stimulated agents for aged people. In addition to nutritional benefits, consumption of donkey's milk has been associated with improved immune responses.

\section{CONCLUSION}

In conclusion, our promising preliminary experiments demonstrated that donkey's milk is an immune-potentiation and to a higher extent of pro-inflammatory cytokine response special in aged subjects. These findings suggest that donkey's milk might be investigated as a beneficial dietary component for up-regulating the immune response in aged people.

\section{ACKNOWLEDGMENTS}

This study was supported by Grant (156-9) from the Student Research Committee, Mazandaran University of Medical Sciences, Sari, Iran.

\section{COMPETING INTERESTS}

The funding organization(s) played no role in the study design; in the collection, analysis and interpretation of data; in the writing of the report; or in the decision to submit the report for publication.

\section{REFERENCES}

Amati L, Marzulli G, Martulli M, Tafaro A, Jirillo F, Pugliese $\mathrm{V}$, et al. Donkey and goat milk intake and modulation of the human aged immune. Curr Pharm Des. 2010;16(7):864-869.

Businco L, Giampietro PG, Lucenti P, Lucaroni F, Pini C, Di Felice G, et al. Allergenicity of mare's milk in children with cow's milk allergy. J Allergy Clin Immunol. 2000;105(5): 1031-1034.

Carroccio A, Cavataio F, Iacono G. Cross-reactivity between milk proteins of different animals. Clin Exp Allergy. 1999;29(8):1014-1016.

Carroccio A, Cavataio F, Montalto G, D'amico D, Alabrese L, Iacono G. Intolerance to hydrolysed cow's milk proteins in infants: clinical characteristics and dietary treatment. Clin Exp Allergy. 2000;30(11):1598-1603.

Criscione A, Cunsolo V, Bordonaro S, Guastella AM, Saletti R, Zuccaro A, et al. Donkeys' milk protein fraction investigated by electrophoretic methods and mass spectrometric analysis. Int Dairy J. 2009;19(4):190-197.

Das S, Bordoloi R, Newar N. A review on immune modulatory effect of some traditional medicinal herbs. J Pharm Chem Biol Sci. 2014;2(1):33-42. 
Gastaldi D, Bertino E, Monti G, Baro C, Fabris C, Lezo A, et al. Donkey's milk detailed lipid composition. Front Biosci. 2010;2(1):537-546.

Ginaldi L, Loreto MF, Corsi MP, Modesti M, De Martinis M. Immunosenescence and infectious diseases. Microbes Infec. 2001;3(10):851-857.

Jirillo E, Candore G, Magrone T, Caruso C. A scientific approach to anti-ageing therapies: state of the art. Curr Pharm Des. 2008;14(26):2637-2642.

Jirillo F, Jirillo E, Magrone T. Donkey's and goat's milk consumption and benefits to human health with special reference to the inflammatory status. Curr Pharm Des. 2010;16(7):859-863.

Jirillo F, Magrone T. Anti-inflammatory and anti-allergic properties of donkey's and goat's milk. Endocr Metab Immune Disord Drug Targets. 2014;14(1):27-37.

Linton PJ, Lustgarten J, Thoman M. T cell function in the aged: Lessons learned from animal models. Clin Appl Immunol Rev. 2006;6(2):73-97.

Lionetti L, Cavaliere G, Bergamo P, Trinchese G, De Filippo C, Gifuni G, et al. Diet supplementation with donkey milk upregulates liver mitochondrial uncoupling, reduces energy efficiency and improves antioxidant and antiinflammatory defences in rats. Mol Nutr Food Res. 2012;56(10):1596-1600.

Mao X, Gu J, Sun Y, Xu S, Zhang X, Yang H, et al. Antiproliferative and anti-tumour effect of active components in donkey milk on A549 human lung cancer cells. Int Dairy J. 2009;19(11):703-708.

Miranda G, Mahé MF, Leroux C, Martin P. Proteomic tools to characterize the protein fraction of Equidae milk. Proteomics. 2004;4(8):2496-2509.

Mocchegiani E, Malavolta M. NK and NKT cell functions in immunosenescence. Aging Cell. 2004;3(4):177-184.

Monti G, Bertino E, Muratore MC, Coscia A, Cresi F, Silvestro L, et al. Efficacy of donkey's milk in treating highly problematic cow's milk allergic children: an in vivo and in vitro study. Pediatr Allergy Immunol. 2007;18(3):258-264.

Pilla R, Dapra V, Zecconi A, Piccinini R. Hygienic and health characteristics of donkey milk during a follow-up study. J Dairy Res. 2010;77(4):392-397.

Piovesana S, Capriotti AL, Cavaliere C, La Barbera G, Samperi R, Chiozzi RZ, et al. Peptidome characterization and bioactivity analysis of donkey milk. J Proteom. 2015;119: 21-29.

Sadiq MA, Tijjani AN, Auwal MS, Mustapha AR, Gulani I. Serological prevalence of brucellosis among donkeys (Equus asinus) in some local government areas of Yobe State, Nigeria. J Equine Vet Sci. 2013;33(3):150-154.

Salimei E, Fantuz F, Coppola R, Chiofalo B, Polidori P, Varisco G. Composition and characteristics of ass's milk. Animal Res. 2004;53(1):67-78.

Tafaro A, Magrone T, Jirillo F, Martemucci G, D’alessandro AG, Amati L, et al. Immunological properties of donkey's milk: its potential use in the prevention of atherosclerosis. Curr Pharm Des. 2007;13(36):3711-3717.

Tidona F, Sekse C, Criscione A, Jacobsen M, Bordonaro S, Marletta D, et al. Antimicrobial effect of donkeys' milk digested in vitro with human gastrointestinal enzymes. Int Dairy J. 2011;21(3):158-165.

Uniacke-Lowe T, Huppertz T, Fox PF. Equine milk proteins: chemistry, structure and nutritional significance. Int Dairy J. 2010;20(9):609-629.

Vincenzetti S, Polidori P, Mariani P, Cammertoni N, Fantuz F, Vita A. Donkey's milk protein fractions characterization. Food Chem. 2008;106(2):640-649.

Zhang XY, Zhao L, Jiang L, Dong ML, Ren FZ. The antimicrobial activity of donkey milk and its microflora changes during storage. Food Control. 2008;19(12):1191-1195.

Received for publication on $09^{\text {th }}$ June 2018 Accepted for publication on $03^{\text {rd }}$ August 2018 